\title{
Curly Flagellar Mutants in Salmonella
}

\author{
By T. IINO \\ National Institute of Genetics, Misima, Japan
}

(Received 1 June 1961)

\begin{abstract}
SUMMARY
A curly flagellar mutant obtained from a strain of Salmonella typhimurium was unstable and repeatedly dissociated 'curly' and normal subclones. Examination of flagellar antigens of the normal and the curly flagellar subclones demonstrated that the changes in flagellar shape corresponded exactly with phase variation: subclones with curly flagella were always in phase-1 (antigen- $i$ ), those with normal flagella were in phase-2 (antigen-1,2). In transduction from a normal flagellar strain to the curly phase-1 strain, transductional clones with normal flagella were isolated. The transductional clones showed the antigen of the donor in phase-1 and that of the recipient in phase-2. This indicates that the phase-1 curly determinant is closely associated with the phase-1 antigen type determinant, $\boldsymbol{H}_{1}$.

Seven curly mutants were obtained from a strain of Salmonella abortus-equi: one with antigen- $a$ (phase-1), and four with antigen-e,n,x (phase-2), and two with antigen-1,2 (phase-2) from a transductional recombinant given antigen-1, 2. Transductional analysis with these strains showed that the phase-2 curly determinant is closely associated with the phase-2 antigen type determinant, $\boldsymbol{H}_{2}$; and the phase-1 curly determinant with $H_{1}$. In cross-absorption experiments with antisera prepared against flagella of either normal or curly mutant, no antigenic difference between normal and curly flagella could be detected. It is inferred that $H_{1}$ and $H_{2}$ are the primary structural determinants of flagellar protein in phase- 1 and phase-2 respectively; mutation in $H_{1}$ or $H_{2}$ may cause an altered configuration of flagellar protein, resulting in a change in antigenic type, or in flagellar shape, or it may cause the failure of flagellar morphogenesis. Attempts to obtain recombination by transduction between the curly flagellar determinants in each of the phases have been unsuccessful; this suggests that in each phase the mutant sites of the curly types are very closely linked or identical.
\end{abstract}

\section{INTRODUCTION}

The shape of bacterial flagella is conditioned by environmental and inherent factors. In cases where the effect of environment is not remarkable, their inherent characteristic shapes are regarded as useful traits for the identification of bacterial species (Leifson \& Hugh, 1953; Leifson \& Palen, 1955). Several mutant types have been described. For instance, mutants with flagella having a wavelength half that found in the wild-type have been discovered in some strains of Proteus (Leifson, Carhart \& Fulton, 1955), Salmonella (Leifson \& Hugh, 1953) and Chromobacterium (Leifson, 1956). This kind of mutant has been called 'curly'.

Recently, an investigation on flagellar regeneration (Kerridge, 1959) demon- 
strated that curly flagella were produced when regeneration occurred in a medium containing $p$-fluorophenylalanine in place of phenylalanine, and that bacteria with such abnormal flagella were almost entirely non-motile. Bacterial flagella are composed of a fibrous protein called flagellin (Astbury, Beighton \& Weibull, 1955). The regeneration experiment suggests that the incorporation of an abnormal amino acid during the synthesis of flagellin causes an alteration of flagellar curvature. If a similar biochemical mechanism is involved in the production of curly flagella of genetic origin, it might be taken as a unique character for the investigation of gene control of protein synthesis, including the coding problem. The genetic analysis of curly flagellar mutants reported in the present paper was undertaken with such expectation.

\section{METHODS}

The salmonella strains mainly used in the present work were Salmonella typhimurium strain SW 577 and S. abortus-equi strains SL 28 and SJ 25. SW 577 is the strain reported by Leifson \& Hugh (1953) as a curly flagellar strain. Its flagellar morphology and clonal instability were first described by them. SL 23 is a phase-2 (antigen- $e, n, x$ ) stable strain received from the Lister Institute of Preventive Medicine, London, by the courtesy of Dr B. A. D. Stocker. SJ 25 is a stable 1, 2 (phase-2) type recombinant obtained by transduction of the $\mathrm{H}_{2}$ gene from $S$. typhimurium TM2 to SL23 (Iino, 1961 a).

Transduction was carried out with phage PLT22 grown on the indicated host cultures. The general procedures of cultivation and transduction were according to the methods of Stocker, Zinder \& Lederberg (1953) and Lederberg \& Iino (1956). Bacterial motility was observed by darkground microscopy of hanging drops of broth. Clonal motility of bacteria was examined on semisolid medium (Stocker et al. 1953). The staining of flagella was by the method of Leifson (1951).

Specific flagellar antisera were prepared in the way described by Edwards \& Ewing (1955). Antigen type was identified qualitatively by slide agglutination tests with antisera of titre 100, and when necessary qualitatively by tube agglutination (Kauffmann, 1954).

\section{RESULTS}

Association of wavelength interchange with phase variation in Salmonella typhimurium $S W 577$

When a broth culture of strain SW 577 is spread on semisolid media, it produces both compact colonies and swarms. Under the microscope in a hanging drop preparation, the bacteria from compact colonies were seen to rotate and aggregate together, while those from swarms move around and disperse. From the stained preparations it was found that the compact colonies were composed of bacteria with curly flagella (wavelength; $1.1 \pm 0.1 \mu$ : amplitude; $0.4 \pm 0.05 \mu$ ) and the swarms of bacteria with normal flagella (wavelength; $2 \cdot 2 \pm 0 \cdot 1 \mu$ : amplitude; $0 \cdot 5 \pm 0 \cdot 05 \mu$ ).

The subclones from a compact colony or a swarm dissociate repeatedly; that is, SW 577 is composed of two types of bacteria: those with normal flagella and those with curly flagella, and the two types interchange. The frequencies of the inter-changes between the two types are of the order of $10^{-4} /$ bacterium/division. This frequent to and fro change of flagellar shape in a culture resembles the phase variation in 
flagellar antigen type (Stocker, 1949; Lederberg \& Iino, 1956). Examination of the flagellar antigen of the normal and the curly clones demonstrated that the change in flagellar shape corresponded exactly with phase variation: in strain SW 577, clones with curly flagella were always in phase- 1 (antigen-i) and clones with normal flagella were always in phase-2 (antigen-1,2) (Table 1).

\section{Table 1. Flagellar characters of 50 subclones derived from a broth culture of} Salmonella typhimurium $S W 577$

\begin{tabular}{|c|c|c|c|c|}
\hline $\begin{array}{l}\text { Number of } \\
\text { subclones }\end{array}$ & $\begin{array}{l}\text { Flagellar } \\
\text { shape }\end{array}$ & $\begin{array}{c}H \text { antigen } \\
\text { type }\end{array}$ & Motility & $\begin{array}{c}\text { Cells in } \\
\text { liquid culture }\end{array}$ \\
\hline $\begin{array}{l}26 \\
24\end{array}$ & $\begin{array}{l}\text { Curly } \\
\text { Normal }\end{array}$ & $i_{1,2}^{i}$ & $\begin{array}{l}\text { Rotation } \\
\text { Translation }\end{array}$ & $\begin{array}{l}\text { Aggregate } \\
\text { Disperse }\end{array}$ \\
\hline
\end{tabular}

Stability of curly character

The curly flagellar type in the $i$-phase of SW 577 rarely reverted to normal flagellar type. Because of the deficient motility of bacteria with curly flagella, such revertants could be detected as swarms on a plate of semi-solid medium containing anti-1, 2 serum on which both curly $i$ and normal 1,2 clones produced only compact colonies. The reverted clones consisted of bacteria with flagella of normal wavelength, and were actively motile in both phases. The reversion rates to normal flagellar type from the curly phase-1 in strain SW 577 and in all the other curly mutants which will be described below were lower than $10^{-6} /$ division. So long as the curly genotype was maintained, the phenotypic expression in a curly flagellar phase was not modified under wide range of cultural conditions investigated: temperatures from $5^{\circ}$ through $40^{\circ}$; in the range from $\mathrm{pH} 5.5$ to 8.5 ; in broth, on nutrient agar or semisolid plates and in Davis' minimal or complete media (Lederberg, 1950). Staining of flagella by the method of Leifson gave convincing and reproducible figures of normal or curly types.

\section{The genetic locus of a curly flagellar determinant}

Transduction was carried out from a phase-2 culture of Salmonella abony (Hantigens $b: e, n, x)$ strain CDC-103 to a phase-1 culture of SW577 (curly-i: 1, 2). Transductional clones producing rapidly spreading swarms on semi-solid plates containing anti-1,2 serum were isolated. The antigen type and the shape of flagella of these isolated transductional clones are listed in Table 2. Among 100 clones tested, 42 had received the phase-2 antigen determinant of the donor, $\mathrm{H}_{2}{ }^{\text {enx }}$, but in phase-1 showed the same antigen, $i$, and curly flagella as the recipient; 56 clones had received both the phase-1 antigen determinant $H_{t}{ }^{b}$ and the determinant of the normal flagellar wavelength from the donor strain. The remaining two clones had acquired normal wavelength flagella, but neither of the $\mathrm{H}$-antigens of the donor. These results indicate that the flagellar shape determinant was closely associated with $H_{1}$. The production of five $i: 1,2$ swarms in the control cultures suggests that two clones of the same type obtained from the transductional experiment were revertants of the curly flagella determinant, rather than recombinants between the antigen type determinant and the flagellar shape determinant. 
Transduction was next carried out from a phase-1 culture of SW 577 to a phase-1 culture of an $A h_{1}$-strain, Salmonella typhimurium SW 1061 (Iino, 1958, 1961 b). $A h_{1}$ is a controller of $H_{1}$-activity and is closely linked to $H_{1}$; a strain which carries $A h_{1}^{-}$ cannot produce flagella, and consequently is non-motile, while it is in phase-1, but when in phase-2 it produces flagella and is normally motile. If the $A h_{1}+$ gene of SW 577 can be transduced separately from the curly determinant, normal $i$-type recombinants will be produced, and can be detected as swarms on semi-solid plates containing anti-1,2 serum. The result of transduction confirmed this expectation: 41 normal $i$-type recombinants grew from the transduction mixture, whereas only one grew in the control plates, on which the same amount of the SW 1061 cultures was incubated without the phage lysate of SW 577. It indicates that $A h_{1}$ and the determinant of the curly flagella were separable by recombination, although $A h_{1}$ was closely linked to $H_{1}$ with which the curly phase-1 determinant is closely associated.

Table 2. Transduction from a phase-2 culture of Salmonella abony $(b: e, n, x)$ to a phase-1 culture of S. typhimurium $S W 577$ (curly-i:1,2)

Recombinants were selected for on plates of semisolid medium containing anti-1,2 serum. Donor: $2 \times 10^{8}$ phage particles. Recipient: $5 \times 10^{7}$ bacteria.

$\begin{array}{lccc} & \begin{array}{c}\text { Flagellar } \\ \text { antigens } \\ b: e, n, x\end{array} & \begin{array}{c}\text { Flagellar } \\ \text { shape }\end{array} & \begin{array}{c}\text { Number of } \\ \text { clones }\end{array} \\ \begin{array}{l}\text { Donor } \\ \text { Recipient }\end{array} & \begin{array}{c}\text { Normal } \\ \text { Curly in } \\ \text { phase-1 }\end{array} & - \\ \text { Recombinants } & \left\{\begin{array}{l}i: e, n, x \\ \text { Curly in } \\ \text { phase-1 }\end{array}\right. & - \\ & \begin{array}{c}\text { Normal } \\ \text { Normal }\end{array} & \mathbf{4 2} \\ \text { Revertants from } & i: 1,2 & \text { Normal } & \mathbf{2} \\ \text { control cultures } & & & \mathbf{5}\end{array}$

Curly flagella in phase-1 of Salmonella abortus-equi SL 23

Salmonella abortus-equi is a serotype which is generally stable in phase-2, antigen$e, n, x$, and carries $V h_{2}-$, a gene which prevents the normal change of flagellar antigenic phase (Iino, 1958, 1961 $a$ ). The hidden phase-1, $a$-antigen type, is obtained by antiserum selection of rare $H_{2}$-inactive bacteria or by the introduction of an inactive $H_{2}$ gene by transduction. Transduction was carried out from a phase-1 culture of $S$. typhimurium TM2 $(i: 1,2)$ to a phase-2 culture of $S$. abortus-equi SL $23(a: e, n, x)$ on semisolid media containing anti-e, $n, x$ serum, in the expectation of obtaining a stable $a$-type by the incorporation of an inactive $\mathrm{H}_{2}{ }^{1,2}$ gene. Recombinant swarms were detected after prolonged (3 days) incubation. Two among 35 swarms thus obtained spread normally. The remaining 33 spread very slowly and formed compact swarms. Flagellar shape was examined on the clones derived from both the normal and the compact swarms after staining by Leifson's method. The clones from the normal swarms were composed of bacteria with normal flagella while those from the compact swarms were composed of bacteria with curly flagella. The curvatures (wavelength and amplitude) of the normal and the curly flagella in SL 23 are the same as those in SW 577. One difference between the two curly types was that in liquid media phase-1 cells of SL 23 did not aggregate as remarkably 
as those of SW 577; consequently, the former produced compact swarms instead of compact colonies on a semisolid plate.

Normal flagellar character was transduced from TM2 to a curly- $a$ transductional derivative of SL 23. The 20 rapidly spreading swarms obtained on semisolid plates were all $i$-type. From these results it is inferred that, like SW 577, SL23thas curly flagella in phase-1 and normal in phase-2 and that the curly,determinant in phase-1 is closely associated with $H_{1}$.

\section{Curly flagella in phase-2}

Bacteria with curly flagella spread very slowly through semisolid medium. Therefore, when a culture with normal flagella is plated on such a medium, a curly mutant is expected to be detected as a dense spot on the faint sheet of motile bacteria. By the use of this method, 63 independently-occurring mutants which did not spread or spread very slowly were isolated from a stable $e, n, x$-type culture of SL 23. Four of them, SJ 30, SJ 163, SJ 164, and SJ 165, were identified to be curly mutants. Four others were paralysed mutants, and the remaining 55 were $\mathrm{Fla}^{-}$. The same

\section{Table 3. Transductions from the strains of Salmonella with normal flagella to those with curly flagella in phase-2}

\begin{tabular}{|c|c|c|c|c|c|}
\hline \multirow{2}{*}{\multicolumn{2}{|c|}{$\begin{array}{l}\text { All of the recipient strains are phase-2 } \\
\text { were selected for on plates of semisolid medi } \\
\text { flagella in phase-2 could produce swarms. S } \\
\text { by revertants of curly flagellar determinant } \\
1 \times 10^{8} \text { bacteria, in each combination. }\end{array}$}} & $\begin{array}{l}\text { opha } \\
\text { n wl } \\
\text { on } \\
\text { lor: }\end{array}$ & $\begin{array}{l}\left.h_{2}^{-}\right) \mathrm{t} \\
\text { ly the } \\
\text { ntrol c } \\
\text { phage } \\
\text { n type }\end{array}$ & warn & $\begin{array}{l}\text { lants } \\
\text { rmal } \\
\text { uced } \\
\text { ient: } \\
\text { n }\end{array}$ \\
\hline & & \multicolumn{2}{|c|}{$\begin{array}{l}\text { Transduction } \\
\text { mixture }\end{array}$} & \multicolumn{2}{|c|}{$\begin{array}{l}\text { Control } \\
\text { culture }\end{array}$} \\
\hline Donor & Recipient & 1,2 & $e, n, x$ & 1,2 & $e, n, x$ \\
\hline $\begin{array}{l}\text { Phase-2 culture } \\
\text { of TM2 }(i: 1,2)\end{array}$ & $\begin{array}{l}\text { SJ30 curly-e, } n, x \\
\text { SJ163 curly-e, } n, x \\
\text { SJ164 curly-e, } n, x \\
\text { SJ } 165 \text { curly-e, } n, x\end{array}$ & $\begin{array}{r}113 \\
75 \\
80 \\
108\end{array}$ & $\begin{array}{r}4 \\
11 \\
2 \\
6\end{array}$ & $\begin{array}{l}\mathbf{0} \\
\mathbf{0} \\
\mathbf{0} \\
\mathbf{0}\end{array}$ & $\begin{array}{r}6 \\
19 \\
4 \\
11\end{array}$ \\
\hline SL23 $(: e, n, x)$ & $\begin{array}{l}\text { SJ167 curly-1,2 } \\
\text { SJ } 168 \text { curly-1,2 }\end{array}$ & $\begin{array}{r}16 \\
2\end{array}$ & $\begin{array}{r}109 \\
90\end{array}$ & $\begin{array}{r}18 \\
2\end{array}$ & $\begin{array}{l}\mathbf{0} \\
\mathbf{0}\end{array}$ \\
\hline
\end{tabular}

selection method was applied to a 1,2-type culture of SJ 25, which is a recombinant obtained by the transduction of the $\mathrm{H}_{2}$ gene from Salmonella typhimurium strain TM2 to SL 23, and two curly mutants, SJ167 and SJ168, were isolated from it. The transduction of the determinant of normal flagellar shape to these curly mutants was always associated with the transfer of the phase-2 antigen determinant $H_{2}$ (Table 3). One remarkable feature of these transductions is that trails were not produced at all on the selective media (see Lederberg, 1956; Stocker, 1956). This suggests that the normal type is not completely dominant over the curly type.

As both SL 23 and SJ 25 are stable phase-2 monophasic types, each carrying a hidden curly phase-1 determinant, no direct evidence has been obtained to exclude the possibility that the newly acquired mutant genes may affect the flagellar shape in phase-1 as well as in phase-2. Nevertheless, it is probable, by analogy with the curly determinant in phase- 1 , that the function of these phase-2 curly determinants is also phase-specific. 


\section{Flagellar antigens of the curly mutants}

The close association of the curly flagella determinants and the $\mathrm{H}$-antigen type determinants in each phase urged us to the detailed examination of the antigenicity of normal and curly flagella. Anti- $i$ sera were obtained against both TM2 (normal- $i$ flagella) and SW 577 (curly- $i$ flagella), and the agglutination titres of the anti- $i$ sera to the $i$-phases of these strains were examined before and after reciprocal absorption (Table 4). The results showed that each serum had the same titre on both normal- $i$ (TM2) and curly-i (SW 577) suspensions, and that each serum could be completely absorbed by the heterologous suspension. That is, the $i$-antigens of curly- $i$ and normal- $i$ flagella behave identically in the serum agglutination experiment. Parallel results were obtained with SL23 (normal-e, $n, x$ flagella) and one of its curly mutants, SJ 30 (curly-e, $n, x$ flagella).

Table 4. Tube agglutination test of the $H$-antigens in normal and curly flagella

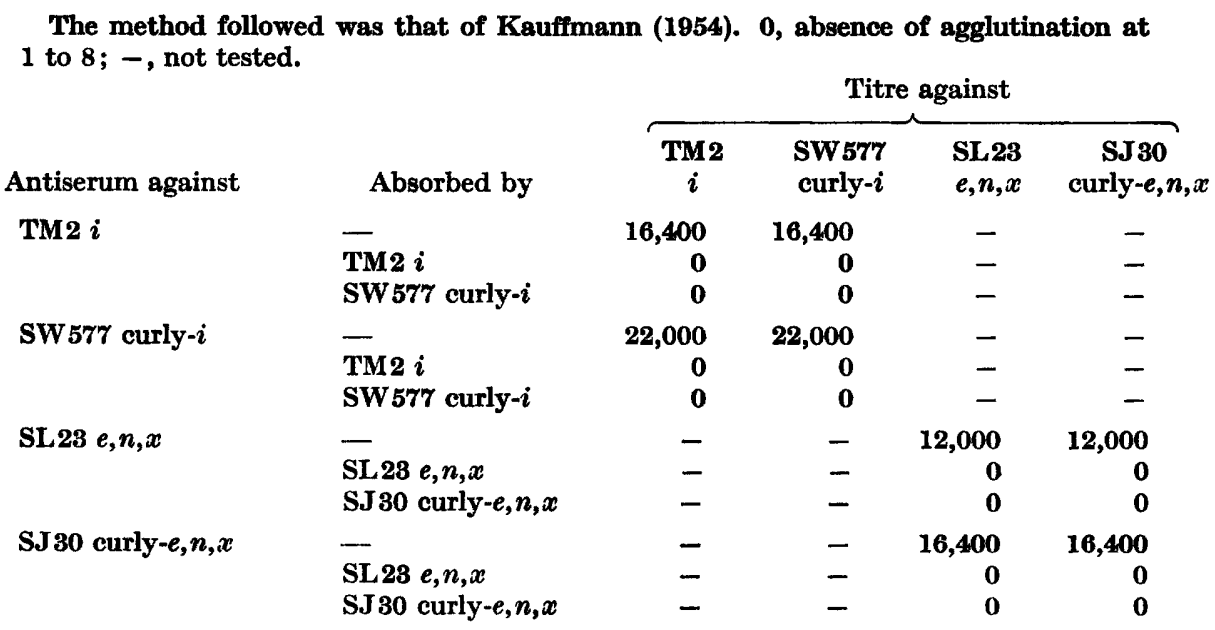

\section{Transductions between curly flagellar mutants}

All the curly flagellar mutants so far obtained revert to the normal type at frequencies which would obscure a low frequency of transduction. Consequently, it is difficult to obtain clear evidence of recombination between curly type determinants of two strains with the same antigen type. In cases where the antigen types of the two mutants are different, we may conclude that a normal type which has the H-antigen of the donor strain in transduction is produced by a recombination between two curly flagella determinants.

In order to get the recombination between the curly flagellar determinant, transduction was carried out from curly- $i$ type of SW 577 to curly- $a$ type of SL23, and from curly-e, $n, x$ type of SJ 30 to curly-1,2 type of either SJ167 or SJ168. In the first combination, a phase-2 culture was used for the preparation of lysate and the transduction of $H_{2}{ }^{1,2}$ was scored as a control: when a phase-2 culture is used as a donor, transduction of $H_{1}$ and $H_{2}$ are expected to be equally common (Lederberg 
\& Iino, 1956). In transductions from SJ 30 (curly-e, $n, x)$, SL23 (normal-e, $n, x$ ) was used as the donor in control experiments. So far, recombinants with normal flagella and with the $\mathrm{H}$-antigens of the donor have not been obtained from any combination, while in each control combination over 100 transductional recombinants were obtained. This suggests that the mutant sites of the two phase-1 curly types are very closely linked or identical; and similarly that the sites of the three phase-2 curly mutants are very closely linked or identical.

\section{DISCUSSION}

The present investigation demonstrated that curly flagellar shape is $\mathbf{H}$-antigen phase-specific in salmonella. The frequent dissociation of a diphasic strain of Salmonella typhimurium SW 577 to normal and curly flagellar types (Leifson \& Hugh, 1953) represents antigenic phase variation. In addition to SW 577, Leifson (1951) described the dissociation of normal and curly flagellar types in another salmonella strain, $S$. wichita SJ64. Serological examination of the dissociated subclones of this strain has now shown that the two curvature types are likewise associated with antigenic phases: curly in phase-1 ( $d$-antigen) and normal in phase-2

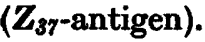

Genetic determinants of the curly flagella are closely associated with the determinants of $\mathrm{H}$-antigen type, $\mathrm{H}_{1}$ and $\mathrm{H}_{2}$ in phase-1 and phase-2 respectively. The flagella of the genus Salmonella are composed of a fibrous protein called flagellin (Astbury et al. 1955; Ambler \& Rees, 1959). The H-antigen type of their flagella is presumed to reflect a specificity of flagellin molecules. The experiment of Kerridge (1959) on flagellar regeneration (see Introduction) suggested that flagellar curvature is also a manifestation of a chemical composition of flagellin. Chi-phage is a strain of bacteriophages which can attack only motile salmonella (Rakieten \& Bornstein, 1941). Like the curly flagellar character, one type of resistance to this bacteriophage has been found to be phase-specific and associated with certain antigen types, antigens $g, \ldots$, which are determined by the $H_{1}$ locus (Meynell, 1961); certain flagellins (or parts of them) are presumably the receptor of this phage. From these primacies of $H_{1}$ and $H_{2}$ in the determination of the specificity of flagella, it is inferred that $H_{1}$ and $H_{2}$ are the primary structural determinants of flagellin; a mutation in one of these genes produces an altered configuration of the corresponding flagellin, presumably through a change in its amino acid sequence, resulting in a change in antigen type, a modification of the flagellar shape or failure of either flagellin synthesis or flagellar morphogenesis.

For the synthesis of flagella, several Fla genes, as well as the $\boldsymbol{H}_{1}$ and $\mathrm{H}_{2}$ genes, are required (Stocker et al. 1953). These Fla genes are however not phase-specific and, as far as investigation has shown, they are not concerned with the specificity of flagella. The only gene which has been known to control a specificity of flagellin in both phases is that which determines the presence of $N$-methyl-lysine in flagellin (Stocker, McDonough \& Ambler, 1961). Its function has been presumed to be not to serve as a primary structural determinant of flagellin but to control the production of a methylator of lysine in already made flagellin molecules.

The failure to obtain normal type recombinants from transductions between curly flagella mutants suggests that in each phase the sites of mutation in the various 
curly type mutants tested are very closely linked or identical. As demonstrated by Kerridge (1959), curly flagella are produced when phenylalanine is replaced by $p$-fluorophenylalanine during flagellar regeneration. Therefore it seems quite possible that the curly flagella of curly mutants result from replacement of a particular amino acid in flagellin by some other; such mutants might occur by mutation of a specific site in $H_{1}$ or $H_{2}$. The comparative chemical analysis of the flagellins from normal and curly-mutant flagella is under investigation.

This investigation was started during the author's stay at the Guinness-Lister Unit, Lister Institute of Preventive Medicine, London, as a visiting investigator from April through July, 1958. The author wishes to express his sincere gratitude to Dr B. A. D. Stocker of the above institute for much helpful advice and discussion. The author is also indebted to Dr E. Leifson (Department of Microbiology, Strich School of Medicine, Loyola University, Chicago, Ill., U.S.A.) for the generous provision of bacterial cultures.

Contribution from the National Institute of Genetics, Japan, No. 387. This work was supported by a research grant from the National Institute of Allergy and Infectious Diseases (E-2872), Public Health Service, U.S.A.

\section{REFERENCES}

Ambler, R. P. \& ReEs, M. W. (1959). $\epsilon$-N-Methyl-lysine in bacterial flagellar protein. Nature, Lond. 184, 56.

Astbury, W. T., Beighton, E. \& Weibull, C. (1955). The structure of bacterial flagella. Symp. Soc. exp. Biol. 9, 282.

Edwards, P. R. \& Ewing, W. H. (1955). Identification of Enterobacteriaceae. Minneapolis, Minn., U.S.A.: Burgess Publ. Co.

Invo, T. (1958). Genetics of phase variation. Abstr. 7th int. Congr. Microbiol. p. 57.

IrNo, T. (1961 a). A stabilizer of antigenic phases in Salmonella abortus-equi. Genetics (in the Press).

IrNo, T. (1961 b). Genetic analysis of O-H variation in salmonella. Jap. J. Genet. 36, 268.

KaUfrmann, F. (1954). Enterobacteriaceae, 2nd ed. Copenhagen: E. Munksgaard.

KERRIDGE, D. (1959). The effect of amino acid analogues on the synthesis of bacterial flagella. Biochim. biophys. acta, 31, 579.

LEDERBERG, J. (1950). Isolation and characterization of biochemical mutants of bacteria. Meth. Med. Res. 3, 5.

LEDERBERG, J. (1956). Linear inheritance in transductional clones. Genetics, 41, 845.

Lederberg, J. \& InNo, T. (1956). Phase variation in salmonella. Genetics, 41, 743.

LEIFson, E. (1951). Staining, shape, and arrangement of bacterial flagella. J. Bact. 62, 377.

LEIrson, E. (1956). Morphological and physiological characteristics of the genus Chromobacterium. J. Bact. 71, 393.

LeIrson, E. \& HuGH, R. (1953). Variation in shape and arrangement of bacterial flagella. J. Bact. 65, 263.

Leirson, E. \& Palen, M. I. (1955). Variation and spontaneous mutation in the genus Listeria in respect to flagellation and motility. J. Bact. 70, 233.

Leiffon, E., Carhart, S. R. \& Fulton, M. (1955). Morphological characteristics of flagella of Proteus and related bacteria. J. Bact. 69, 73.

MeYnel, E. W. (1961). A phage, $\phi \chi$, which attacks motile bacteria. J. gen. Microbiol. $25,253$.

RAkIeten, M. L. \& Bornstein, S. (1941). Influence of certain bacteriophages on the $\mathrm{H}$ antigens of Salmonella poona and E. typhi. Proc. Soc. exp. Biol., N.Y. 48, 359. 
Stocker, B. A. D. (1949). Measurement of rate of mutation of flagellar antigenic phase in Salmonella. J. Hyg., Camb. 47, 308.

STocker, B. A. D. (1956). Abortive transduction of motility in salmonella; a nonreplicated gene transmitted through many generations to a single descendant. J. gen. Microbiol. 15, 575.

Stocker, B. A. D., McDonough, M. W. \& Ambler, R. P. (1961). A gene determining presence or absence of $\epsilon-N$-methyl-lysine in salmonella flagellar protein. Nature, Lond. 189, 556.

Stocker, B. A. D., Zinder, N. D. \& LeDenberg, J. (1953). Transduction of flagellar characters in salmonella. J. gen. Microbiol. 9, 410. 